\title{
Correlation between resistance of Pseudomonas aeruginosa to benzalkonium chloride and expression of efflux pump genes
}

\author{
Mohamed El-Sayed Osman¹, Hoda Hamed El-Hendawy¹, Said Mohamed Abd El-All², Ahmed Mohamed Hassan², \\ Dina Ezzat Mahmoud ${ }^{4 *}$ \\ ${ }^{1}$ Professor of Microbiology, Department of Botany and Microbiology, Faculty of Science, Helwan University, Egypt. \\ ${ }^{2}$ Professor of Microbiology, National Organization for Drug Control and Research (NODCAR), Cairo, Egypt. \\ ${ }^{3}$ Researcher, Department of Microbiology, National Organization for Drug Control and Research (NODCAR), Cairo, Egypt. \\ ${ }^{4}$ Researcher Assistant, Department of Microbiology, National Organization for Drug Control and Research (NODCAR), Cairo, Egypt.
}

\begin{tabular}{l}
\hline ARTICLE INFO \\
\hline Received on: 29/09/2018 \\
Accepted on: 22/10/2018 \\
Available online: $30 / 12 / 2018$ \\
\\
\hline Key words: \\
Pseudomonas aeruginosa, \\
benzalkonium chloride (BC), \\
cross-resistance, efflux pump, \\
efflux pump inhibitors, \\
sertraline.
\end{tabular}

\section{INTRODUCTION}

Disinfectants have a vital role in keeping acceptable health standards by significantly decreasing microbial loads and inactivating pathogens (Antonio et al., 2017). Quaternary ammonium compounds (QACs) are the main biocide (antiseptics and disinfectants) used in various fields such as health care and agriculture as well as in industry (Ebrahimi et al., 2016).

Benzalkonium chloride (BC) is the most important QACs used for surfaces disinfection in medical care applications because of their antibacterial activity (Antunes et al., 2016) in addition to its important use as a preservative in aqueous formulations to prevent several infections (Nones et al., 2017).

\footnotetext{
${ }^{*}$ Corresponding Author

Dina Ezzat Mahmoud, Researcher Assistant, National Organization for Drug Control and Research (NODCAR), Cairo, Egypt.

E-mail:Dina91286@yahoo.com
}

Pseudomonas aeruginosa is considered to be a frequent and severe cause of acute nosocomial infections, especially affecting intensive care unit (ICU) and immune-compromised patients (Cabot et al., 2016).Previous studies suggested that exposure to subinhibitory concentrations of BC leads to co- and cross-resistance to other antimicrobial agents (Rakic-Martinez et al., 2011).

Pseudomonas aeruginosa resists the lethal effects of BC by several mechanisms, such as increased efflux pump activity, mutations in GyrA and ParC genes that encode DNA gyrase and topoisomerase IV in addition to changes in the bacterial membrane (Ferreira et al., 2011).

While there are at least 12 structural genes for the resistance-nodulation-division efflux systems have been identified in $P$. aeruginosa genome, four of them are clinically-important (MexAB-OprM, MexCD-OprJ, MexEF-OprN, and MexXY-OprM) (Lister et al., 2009). Mex systems co-expression has been reported in $P$. aeruginosa clinical isolates where its variable effect on antibiotic susceptibility has been observed (Poonsuk and Chuanchuen, 2014). 
Phenotypic and genetic tests used to identify acquired resistance due to efflux pump overexpression in $P$. aeruginosa isolates. Over-expression of efflux pumps could increase the MICs of many antimicrobials. Different compounds have been known as efflux pump inhibitors (EPIs) due to its ability to broadly inhibit several known multidrug EPs in P. aeruginosa (Adabi et al., 2015). The purpose of this study was the cross-resistance determination of the BC-adapted $P$. aeruginosa with other antimicrobial agents. In addition to phenotypic and genotypic evaluation of the resistance caused by efflux pump activity.

\section{MATERIALS AND METHODS}

\section{Bacterial isolates}

Totally, 88 non-duplicated $P$. aeruginosa isolates were collected from clinical settings at four hospitals, Kasr El-Aini Hospital, Ain-Shams University Hospital, Chest Hospital, and Al Salam Hospital in Cairo, Egypt along the period of January-March 2015. Isolates those were Gram-negative with greenish-yellow colonies, fluorescent-yellow colonies, and blue color colonies on Cetrimide Agar, Pseudomonas Agar F, and Pseudomonas Agar $\mathrm{P}$, respectively. Oxidase, catalase, and nitrate reductase positive isolates were identified as P. aeruginosa (Mahon et al., 2014). Pseudomonas aeruginosa wild-type strain (ATCC 15442) was purchased from Microbiologica USA.

\section{Benzalkonium chloride}

$\mathrm{BC}$ was purchased from Scharlabs S. L., Spain.

\section{Minimum inhibitory concentration (MIC) of benzalkonium chloride}

The MIC of benzalkonium chloride was carried out against the $88 P$. aeruginosa isolates and $P$. aeruginosa strain (ATCC 15442) using agar dilution method due to precipitation of $\mathrm{BC}$ at high concentrations, following Clinical and Laboratory Standards (CLSI) (formerly NCCLS) in document M100-S14 (NCCLS, 2004; Kawamura-Sato et al., 2010). One microliter of each bacterial suspension contained $10^{4} \mathrm{cfu} \mathrm{ml}^{-1}$ was inoculated on Mueller-Hinton agar (Oxoid) with different BC concentrations, then incubated at $37^{\circ} \mathrm{C}$ for 18 hours and the MIC was recorded. The $\mathrm{BC}$ adapted isolates were selected for further investigation.

\section{Antibiotics susceptibility testing (disk diffusion method)}

Antibiotic susceptibility pattern of $\mathrm{BC}$-adapted isolates was determined by the single disk diffusion test (Kirby-Bauer method) (Bauer et al., 1966; CLSI, 2008). According to CLSI (2008), the following antibiotic discs (Oxoid) were used: Gentamicin-CN $(10 \mu \mathrm{g})$, Piperacillin-tazobactam PIP/TAZ $(100 / 10 \mu \mathrm{g})$, Ciprofloxacin-CIP (5 $\mu \mathrm{g})$, Amikacin-AK (30 $\mu \mathrm{g})$, Ceftazidime - CAZ $(30 \mu \mathrm{g})$, Imipenem-IPM $(10 \mu \mathrm{g})$, and Piperacillin-PRL $(100 \mu \mathrm{g})$

\section{Determination of cross-resistance to other antimicrobials}

The MIC of cetrimide (Hopkin \& Williams, UK), iodine (El-Nile Company, Egypt), and ciprofloxacin (ADWIA, Egypt) was determined by the agar dilution method (Kawamura-Sato et al., 2010; NCCLS, 2004), but due to ethanol (Fisher Scientific, UK) evaporation, its MIC was determined by macro-dilution method (Mazzola et al., 2009).

\section{Detection of efflux pump genes by PCR technique}

PCR amplification of the efflux pump regulatory genes (MexR, NfxB, MexT, and MexZ) was performed using primers shown in Table 1 (Linares et al., 2005). The PCR mixture containing $100 \mathrm{ng}$ of chromosomal DNA, $0.5 \mu \mathrm{M}$ concentration of each primer, and Dream Taq ${ }^{\mathrm{TM}}$ Green PCR Master Mix (ThermoFisher Scientific, USA) was heated for 3 minutes at $94^{\circ} \mathrm{C}$. This was followed by 32 cycles of 30 seconds at $94^{\circ} \mathrm{C} ; 30$ seconds at $53^{\circ} \mathrm{C}(\mathrm{NfxB}), 55^{\circ} \mathrm{C}(\mathrm{MexT}), 58^{\circ} \mathrm{C}(\mathrm{MexR})$, or $60^{\circ} \mathrm{C}(\mathrm{MexZ})$; and 60 seconds at $72^{\circ} \mathrm{C}$, with final 10 minutes extension step at $72^{\circ} \mathrm{C}$.

\section{Phenotypic determination of efflux pumps activity}

Ethidium bromide (EtBr)-agar cartwheel method (screening method)

Plates of Tryptic Soy Agar (TSA) containing EtBr concentrations (Sigma Aldrich) ranging from 0 to $5 \mathrm{mg} / \mathrm{l}$ were swabbed with bacterial cultures. Cartwheel pattern was made by dipping a swab into each culture and streaked them from the central circle to the margin of the plate.

EtBr-agar plates were incubated for 16-18 hours at $37^{\circ} \mathrm{C}$. Each EtBr plate was examined under a UV light. The minimal concentration of $\mathrm{EtBr}$ which produced fluorescence of the swabbed culture was recorded for the wild-type strain and $\mathrm{BC}$-adapted isolates. The higher the concentration of $\mathrm{EtBr}$ required for the appearance of fluorescence the greater the presumptive EP activity (Martins et al., 2013).

\section{Sequencing of the efflux pump genes}

PCR products of the selected isolate and $P$. aeruginosa ATCC 15442 strain were purified using the DNA purifying kit

Table 1. Primer sequences used in efflux pump regulatory genes detection.

\begin{tabular}{cccc}
\hline Efflux pumps & Gene & Product size (bp) & Sequence \\
\hline MexAB-OprM & mexR & 637 & Forward: CGCCATGGCCCATATTGAG \\
MexCD-OprJ & nfxB & & Reverse: GGCATTCGCCAGTAAGCGG \\
& & 939 & Forward: CG ATCCTTCCTATTGCACG \\
MexEF-OprN & mexT & 997 & Reverse: GCCAAGTGCCAGTATCG \\
& & & Forward: CGGTTGCAGCCTCTAGCC \\
MexXY-OprM & mexZ & 781 & Forward: AGCGGCGCGACAGTAGCATA \\
& & Reverse: CCGAGGACCAGCGCAGGC \\
\hline
\end{tabular}


(Thermo Fischer, USA) following the manufacturer's instruction. The samples were processed using DNA sequencing kit (Thermo Fischer, USA) and analyzed in an automatic DNA sequencer (Applied Biosystem —-serial no. G: 43 A: 39 T: 30 C: 41) in order to analyze mutation in genes. The DNA sequences were aligned and homology searches were performed using BLAST program (www.ncbi.nlm.nih.gov/blast) and the translation to amino acids was performed using ExPASy translate https://web.expasy.org/ translate/.

\section{Efflux pumps inhibitors (EPIs) (confirmatory method)}

\section{Determination of MIC of EPIS}

The MIC of EPIs (listed in Table 2) has been determined by microtiter method (Wiegand et al., 2008). A $100 \mu$ of broth was added into the negative control well (column 12) and 50 $\mu \mathrm{l}$ into the positive control well (column 11). Fifty microliters of each EPIs dilution (twice the desired final conc.) was added into the respective well (columns 1-10) for each bacterial isolates. The bacterial suspension was adjusted to $1 \times 10^{8} 1$ $\mathrm{ml}^{-1}$ and diluted to $1: 100$. All wells except the negative control were inoculated with $50 \mu \mathrm{l}$ of the bacterial inoculums with final inoculum concentration $5 \times 10^{5} \mathrm{cfu} \mathrm{ml}^{-1}$.

\section{Determination of MIC of BC in combination with EPIS}

The antimicrobial efficacy of BC in combination with EPIs against $\mathrm{BC}$-adapted $P$. aeruginosa was evaluated using the agar dilution method. The concentrations tested typically ranged from 2 to 3 concentration below the MIC of $\mathrm{BC}$ and EPIs. Organisms and agar dilution plates were prepared as for the MIC determination with exception of the addition of $\mathrm{BC}$ and EPIs plus an addition of agar medium to reach the $20 \mathrm{ml}$ of the total (NCCLS, 2004). The fractional inhibitory concentration (FIC) index was calculated to evaluate the effect of the combinations (Satish et al., 2005).

\section{Genotypic determination of efflux pumps activity}

Gene expression analysis of MexA, MexC, MexE, and MexX by real-time PCR

RT-PCR was used to determine the expression of the genes encoding the four major $P$. aeruginosa efflux pumps MexA, MexC, MexE, and MexX of the selected isolate and P. aeruginosa wild-type strain ATCC 15442 (as a control).

Total RNA was extracted by the RNeasy Mini Kit (Qiagen, Germany) following its instructions and QIAGEN RNase-Free DNase Set (Qiagen, Germany) was used to remove the residual DNA. Finally, RNA was dissolved in $50 \mu \mathrm{l}$ of RNase-free water. Synthesis of cDNA was performed by reverse transcription using QIAGEN OneStep RT-PCR Kit (Thermo Fisher). rpsL gene expression was measured as an internal control for confirmation that the amounts of RNA were equal in all of the RT-PCRs. The amplification of the selected genes was performed with specific primers (listed in Table 3 ). The reaction mixtures were incubated at $50^{\circ} \mathrm{C}$ for 30 minutes and 5 minutes at $94^{\circ} \mathrm{C}$. Followed by 40 cycles of 15 seconds at $94^{\circ} \mathrm{C} ; 30$ seconds at $57^{\circ} \mathrm{C}$ (for MexA), $55^{\circ} \mathrm{C}$ (for $\mathrm{MexC}$ and MexE), $60^{\circ} \mathrm{C}$ (for MexX and rpsL); and 1 minute at $72^{\circ} \mathrm{C}$, with final 7 minutes elongation at $72^{\circ} \mathrm{C}$ (Linares et al., 2005).

\section{Analysis of the SYBR green RT-PCR results}

Stratagene MX3005P software was used to determine the amplification curves and $\mathrm{Ct}$ values. The $\mathrm{Ct}$ of each sample was compared with that of the control group according to the " $\Delta \Delta \mathrm{Ct}$ " method to estimate the variation of gene expression on the RNA of the different samples (Yuan et al., 2006).

\section{RESULTS AND DISCUSSION}

Antibiotic resistance in bacterial pathogens has increased worldwide in the last decade leading to failures in the treatment of infectious diseases in human and animals (Gnanadhas et al., 2013).

Table 2. Efflux pumps inhibitors used.

\begin{tabular}{ccc}
\hline Efflux pumps inhibitor & Solvent & Manufacturer company \\
\hline CCCP & Dimethylsulfoxide (DMSO) & Sigma Aldrich (USA) \\
Trimethoprim & $\mathrm{HCl}$ & EL Nasr Co. \\
Epinephrine & $\mathrm{H}_{2} \mathrm{O}$ & MISR Co. Egypt \\
Sertraline & DMSO & Global Pharmaceutical Industries, Egypt \\
\hline
\end{tabular}

Table 3. Primers used in RT-PCR.

\begin{tabular}{ccc}
\hline Gene & Product size (bp) & Sequence \\
\hline mexA & 332 & Forward: CCTGCTGGTCGCGATTTCGG \\
& 390 & Reverse: CCAGCAGCTTGTAGCGCTGG \\
mexC & 260 & Forward: TTGGCTATGGCCATCGCGTT \\
& & Forward: ATCCCACTTCTCCTGGCGCT \\
mexE & 812 & Reverse: GGTCGCCTTTCTTCACCAGT \\
mexX & & Forward: GCGATGCGGATTGCGGAACA \\
& 231 & Reverse: TGGTCGCCCTATTCCTGCTG \\
RPSL* & & Forward: GCAACTATCAACCAGCTG \\
\hline
\end{tabular}

*The ribosomal rpsL gene was used as a housekeeping reference gene. 
Nosocomial infection is a worldwide problem and reported in $7 \%-8 \%$ of in-patients. Pseudomonas aeruginosa is responsible for $6 \%-25 \%$ of nosocomial infections. Treatment of infections caused by $P$. aeruginosa is very complicated because of their remarkable virulence characteristics and frequent antibiotic resistance (Yuruken et al., 2016).

Benzalkonium chloride (BC) is a QAC which have broadspectrum antimicrobial activity (Ebrahimi et al., 2016; McCay et al., 2010). Low and higher level of adaptation to the $\mathrm{BC}$ has been observed in $P$. aeruginosa strains (Langsrud et al., 2003). Adaptation to $\mathrm{BC}$ in $P$. aeruginosa is mediated primarily by the efflux pumps activity, most notably the Mex systems (Lomovskaya et al., 1999). Vijaya et al. (2016) reported that about $22 \%$ of multidrug-resistant $P$. aeruginosa isolates showed insusceptibility to BC.

Determination of minimal inhibitory concentration (MIC) of Benzalkonium Chloride (BC) against Pseudomonas aeruginosa isolates

The MIC of the wild-type strain and $P$. aeruginosa isolates were shown in Table 4, which indicated that 20 isolates were $2,048 \mathrm{mg} / \mathrm{l}, 55$ isolates were $512 \mathrm{mg} / \mathrm{l}$, and 13 isolates, as well as wild-type strain was $256 \mathrm{mg} / \mathrm{l}$. The $20 \mathrm{BC}$-adapted isolates were selected for the further testing.

Lambert and Pearson (2000) reported that the MIC of BC against $P$. aeruginosa isolates was 4,844, 1,462, 346, and 83.7 $\mathrm{mg} / \mathrm{l}$. Also, Walsh et al. (2003) found the MIC of BC against $P$. aeruginosa isolates was $500 \mathrm{mg} / \mathrm{l}$.

\section{Sources of the $20 \mathrm{BC}$-adapted isolate}

The $20 \mathrm{BC}$-adapted isolates were collected from ICU, sputum, pus, urine, and wounds (Fig. 1).

\section{Antibiotics susceptibility screening by Disk diffusion method}

The antibiotics susceptibility of the 20 BC-adapted isolates against the selected antibiotics is shown in Figure 2, which

Table 4. MIC values of BC.

\begin{tabular}{cc}
\hline No. of isolates & MIC value (mg/l) \\
\hline Wild-type strain & 256 \\
13 & 256 \\
55 & 512 \\
20 & 2,048 \\
\hline
\end{tabular}

indicates that all isolates were resistant to at least six antibiotics (multidrug resistant).

Determination of cross-resistance to the other antimicrobial agents

The MIC values of the selected $20 \mathrm{BC}$-adapted isolates and the wild-type strain ATCC 15442 were compared with cetrimide (QAC), ethanol (Alcohol), iodine (halogens), and ciprofloxacin (quinolones) for determination of cross-resistance to the other antimicrobial agents. The obtained results showed that the $20 \mathrm{BC}$-adapted isolates were also adapted to cetrimide with MIC $7,000 \mathrm{mg} / 1$ and ciprofloxacin with MIC was 64 or $128 \mathrm{mg} / \mathrm{l}$, but not adapted to ethanol and iodine. As reported by Buffet-Bataillon et al. (2016), it was found that adaptation to BC leads to cross-resistance with fluoroquinolones.

Detection of efflux pump genes (MexR, NfxB, MexT, and MexZ) by PCR technique

The amplification products of MexR, NfxB, MexT, and MexZ genes were identified by agarose gel electrophoresis with ethidium bromide staining. The amplified products were 637bp fragment for MexR gene, 939-bp fragment for NfxB, 997-bp fragment for MexT gene, and 781-bp fragment for MexZ gene.

MexR gene was detected in 16 isolates, NfxB gene in 18 isolates, MexT gene in 10 isolates, and MexZ gene in 17 isolates (Fig. 3).

The four genes were found collectively in nine isolates (isolates no. 7, 15, 28, 37, 51, 52, 86, 87, and 154), these nine isolates were selected for the further investigation.

\section{Evaluation of the efflux pump activity by Ethidium bromide} (EtBr) cartwheel method

The presumptive identification of the highest EP activity in the nine isolates and the wild-type strain ATCC 15442 was carried out by using the EtBr-agar Cartwheel method. Isolates express efflux pumps by variable levels as shown in Figure 4. The ATCC strain was found to fluoresce at conc. $0.5 \mathrm{mg} / 1$ of EtBr, which means it has no efflux pump activity (negative control).

The minimum concentration at which the adapted isolates showed fluorescence was $1 \mathrm{mg} / \mathrm{l}$ for isolates no. 15,51 , and 154 , while $2.5 \mathrm{mg} / \mathrm{l}$ for isolates no. 28 and 37 , but isolate no. 86 was $4.5 \mathrm{mg} / \mathrm{l}$, isolates no. 7 and 52 were $5 \mathrm{mg} / \mathrm{l}$, however, the isolate no. 87 was more than $5 \mathrm{mg} / \mathrm{l}$. These results were agreed

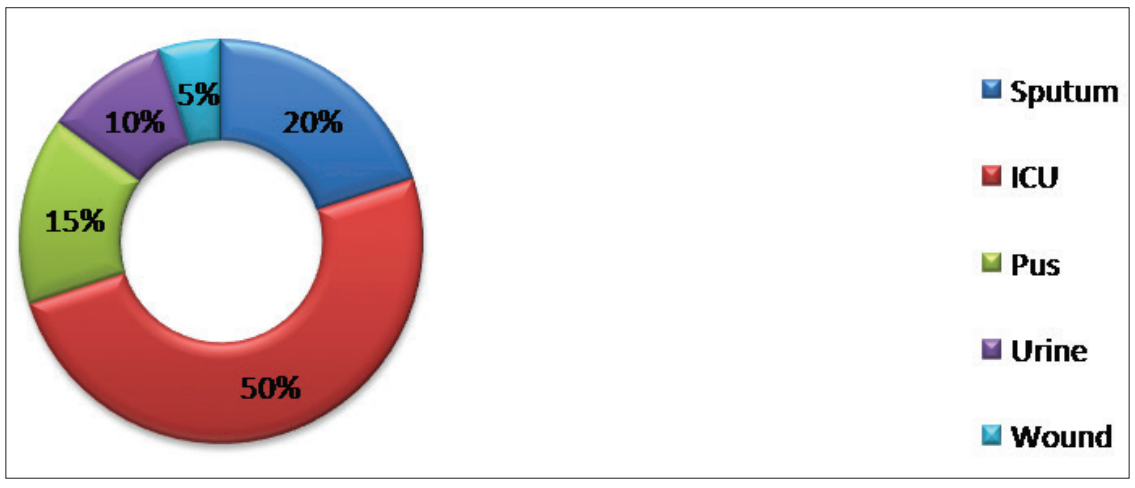

Figure 1. The sources of the BC-adapted isolates. 


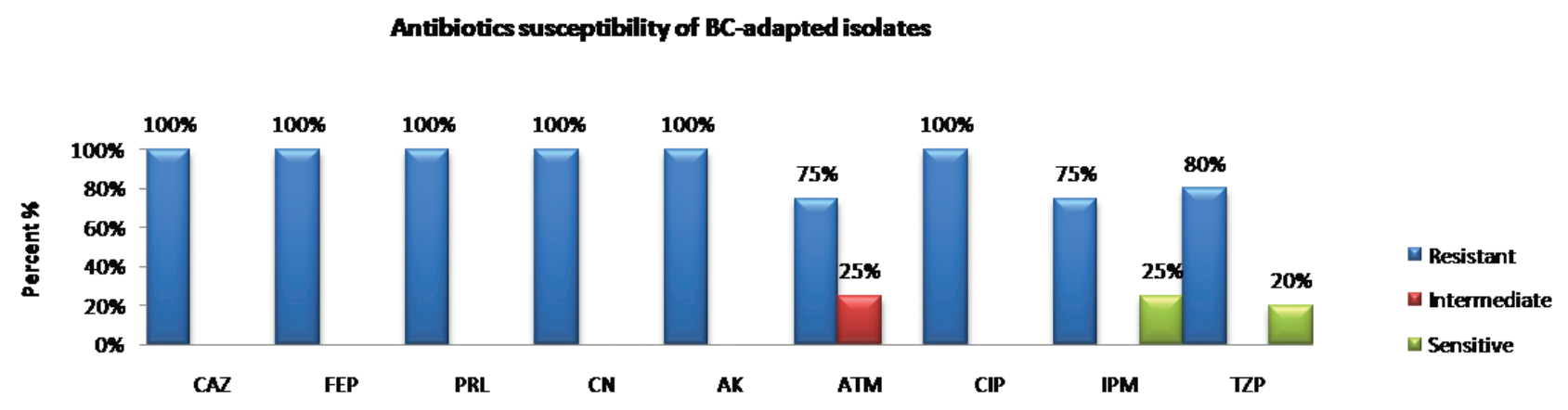

Antibiotics

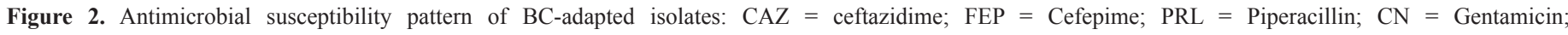
$\mathrm{AK}=$ Amikacin; ATM = Aztreonam; CIP = Ciprofloxacin; IPM = Imipenem; TZP = Piperacillin-tazobactam .

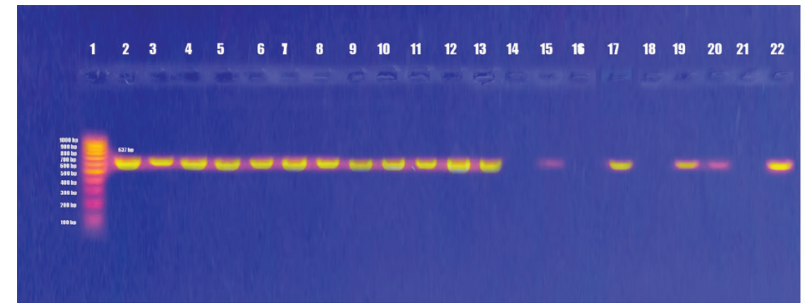

(A) MexR gene

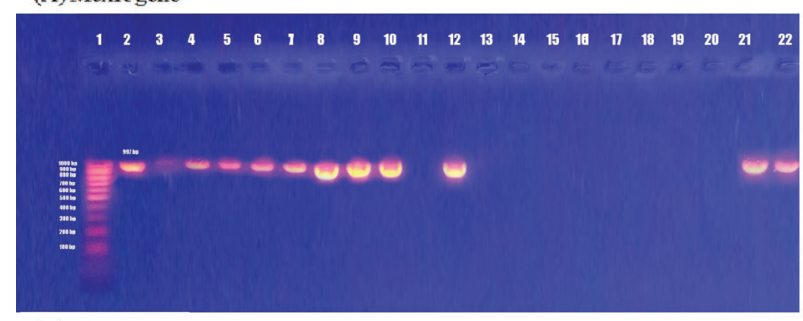

(C) MexT gene

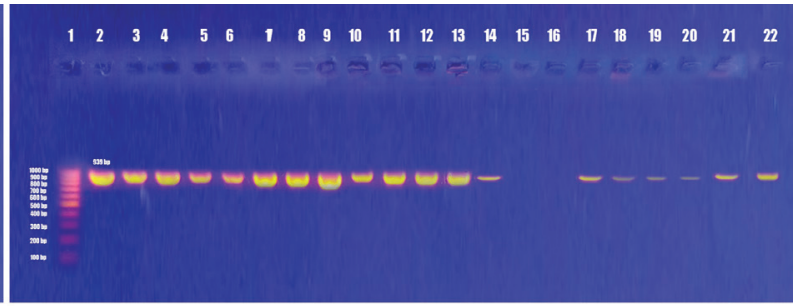

(B) NfxB gene

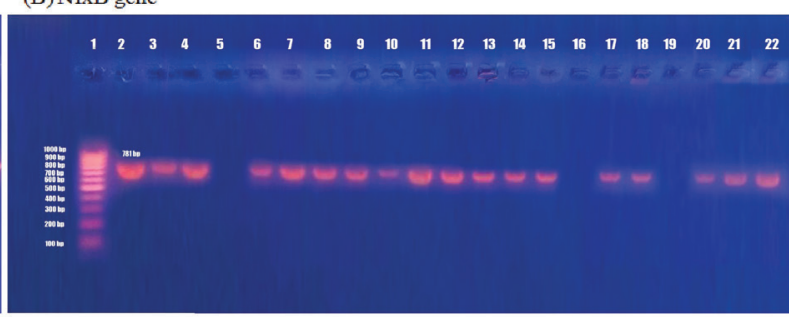

(D) Mex $Z$ gene

Figure 3. Detection of Efflux pump regulatory genes: (A) MexR gene, (B) NfxB gene, (C) MexT gene, and (D) MexZ gene by garose gel electrophoresis of PCRamplified products. Lane 1 represented DNA ladder; lane 2, the amplified products for wild-type strain (ATCC 15442); and lanes from 3 to 22 represent the amplified products for the selected 20 isolates $(52,87,1,7,15,28,37,51,76,86,90,108,116,117,132,134,135,149,153$, and 154, respectively).

with El-Naggar et al. (2011), who reported that EtBr was effluxed in $P$. aeruginosa.

The previous results revealed that isolate no. 87 has a high efflux pump activity.

\section{Sequencing}

To identify mutations, sequences of the selected isolate no. 87 and wild-type strain ATCC 15442 were compared with P. aeruginosa PAO1 (Pseudomonas Genome Database, (http:// www.pseudomonas.com). Figures 5-8 showed the amino acids alignment of efflux pump regulatory genes MexR, NfxB, MexT, and MexZ of wild-type strain PAO1, wild-type strain ATCC 15442, and isolate no. 87, respectively.

As shown in Figures 5-8, in the MexR regulatory gene, a novel mutation in codon number 126 was correlated to resistance to fluoroquinolone, which is substitution of amino acid Valine (V) to Glutamic acid (E), which was reported in previous investigations (Nguyen et al., 2018; Vaez et al., 2018). Results of several independent studies revealed different mutations, for example, Suman et al. (2006) reported 24 silent and four missense mutations in 14 clinical isolates of $P$. aeruginosa, and Savov et al. (2014) found another mutation in codon 44 in one strain, substitution of amino acid lysine to methionine.

No mutations were detected in $\mathrm{NfxB}$ in this study, Esquisabel et al. (2011) found several different amino acid substitutions, while the amino acid substitutions of Asparagine $(\mathrm{N})$ to Glycine $(\mathrm{G})$ in codon 68 and Arginine $(\mathrm{R})$ to Histidine $(\mathrm{H})$ in codon 33 were previously reported in clinical strains of $P$. aeruginosa (Livermore, 2002; Suman et al., 2006).

In MexT, two substitutions were found, Proline (P) to Alanine (A) in codon 81 and Phenylalanine (F) to Isoleucine (I) in codon 172. These mutations were also observed in wild-type strain ATCC 15442 so they considered as a non-significant mutation.

In MexZ, three substitutions were found. One substitution was Leucine (L) to Arginine (R) in codon 138. In addition to two conservative substitutions producing amino acids with similar physiochemical properties [Methionine (M) to Valine (V) in codon 1 and Aspartic acid (D) to Glutamic acid (E) in codon 155]. These 

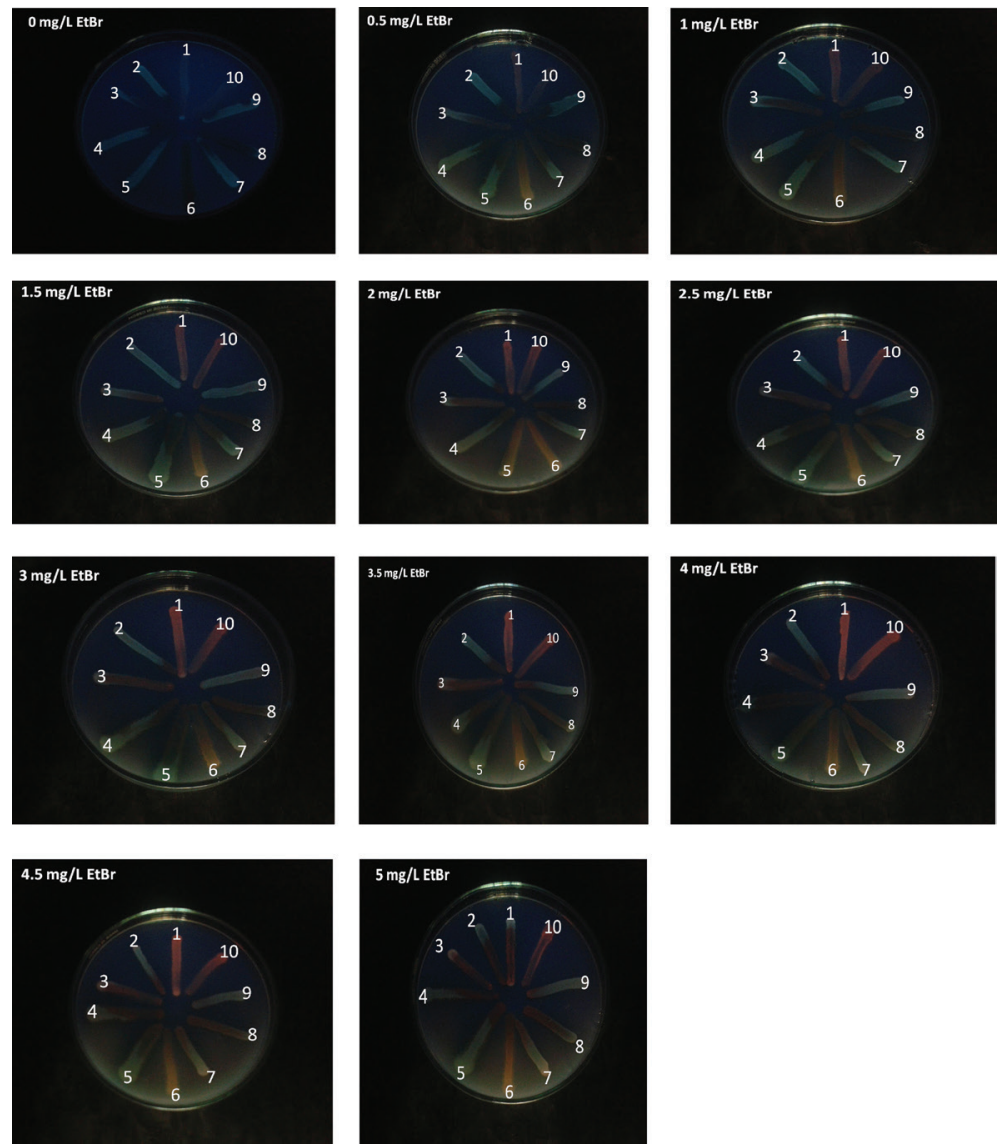

Figure 4. Fluorescence of the isolates on agar plates containing increasing concentrations of EtBr. cultures were swabbed in TSA plates containing increasing concentrations of EtBr. Following overnight incubation at $37^{\circ} \mathrm{C}$ for 16 hours, fluorescence was detected under UV light. No. 1: ATCC 15442, from 2 to 10 refer to isolates no. $7,15,28,37,51,52,86,87$, and 154 , respectively.

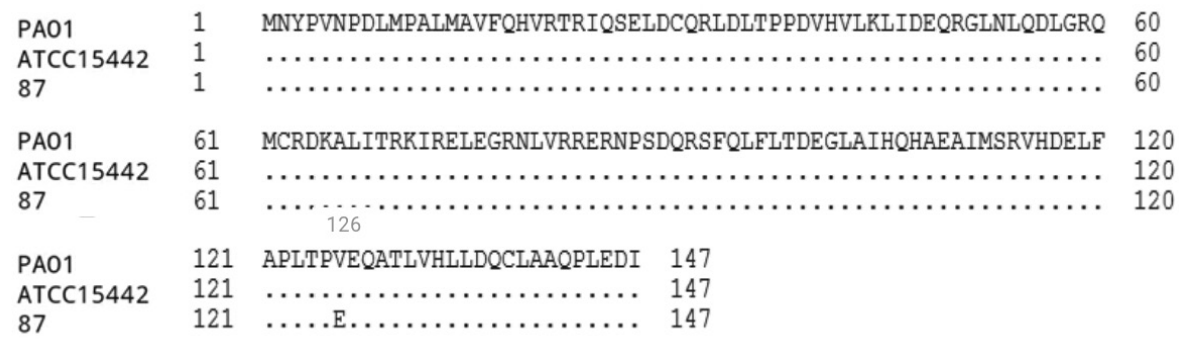

Figure 5. Alignment of amino acid sequences of the MexR gene.

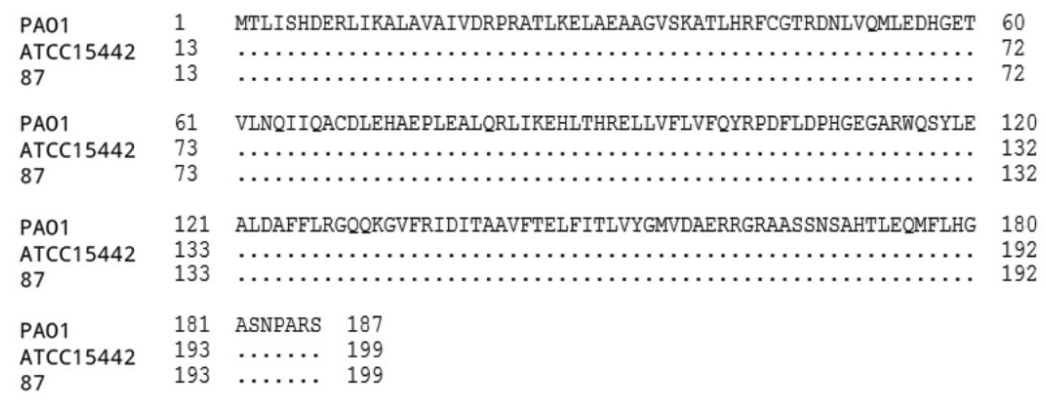

Figure 6. Alignment of amino acid sequences of the NfxB gene. 


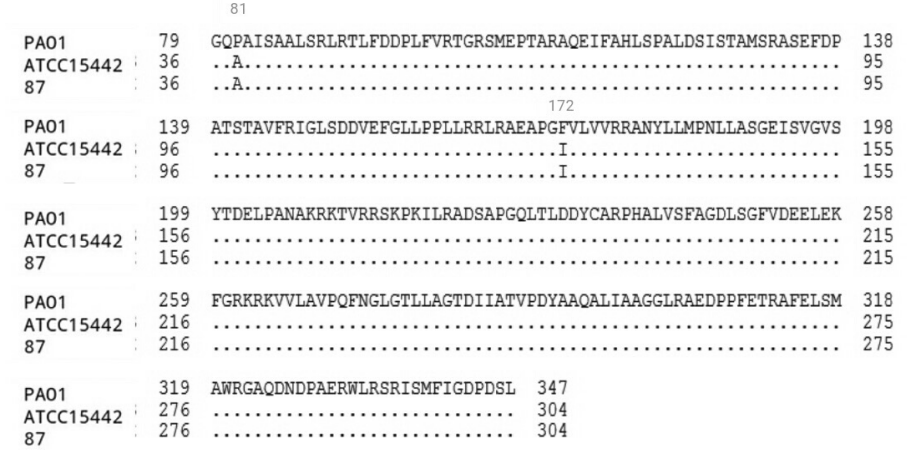

Figure 7. Alignment of amino acid sequences of the MexT gene.

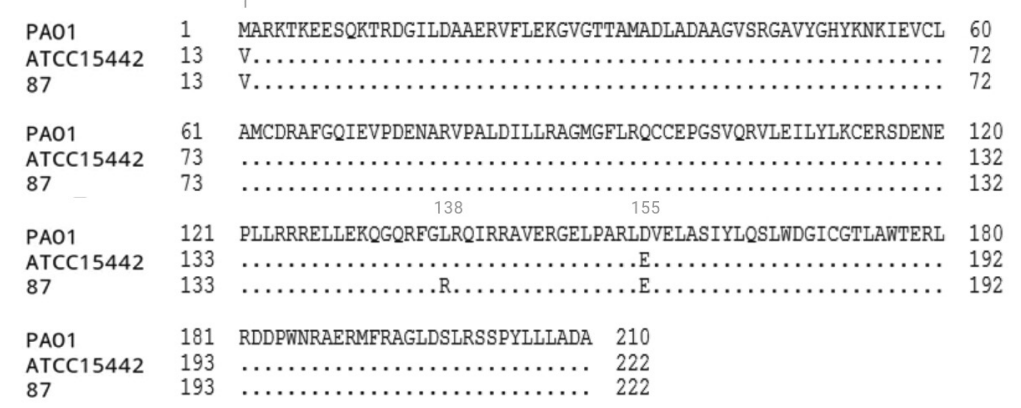

Figure 8. Alignment of amino acid sequences of the MexZ gene.

results were as reported by Nemat-Gorgani (2009). Esquisabel et al. (2011) observed the same substitution, but in another codon (Leu-162-Arg).

\section{Efflux pumps inhibitors (EPIs) (confirmatory method)}

The MIC values of carbonyl cyanide m-chlorophenylhydrazone (CCCP) was $128 \mathrm{mg} / \mathrm{l}$ and MIC value of sertraline was $1,000 \mathrm{mg} / \mathrm{l}$ for both isolates no. 87 and the wildtype strain ATCC 15442. But trimethoprim and epinephrine have no antimicrobial activity.

Efflux pump activity of isolate no. 87 was evaluated in the absence and presence of an efflux pump inhibitors (EPI) used.

According to the obtained results, the insusceptibility of the isolate 87 to $\mathrm{BC}$ decreased upon the combination of $\mathrm{BC}$ with the sertraline (2,048-256 mg/l BC). Sertraline had the most potent antimicrobial activity among the four EPIs used. It was found that the concentration $256 \mathrm{mg} / \mathrm{l}$ of $\mathrm{BC}+800 \mathrm{mg} / \mathrm{l}$ of Ser. is the best concentrations which inhibit the growth of all bacterial isolates. While CCCP, TMP, and EP have no effect.

The obtained results revealed that efflux pumps in isolate 87 play an important role in adaptation development to BC.

Data presented in Table 5 revealed that the FIC index values stated a partial synergistic antimicrobial activity of BC-Ser against all tested bacterial isolates (FIC $>0.5$ but $<1$ ).

Bohnert et al. (2011) reported that resistance of the isolates to $\mathrm{BC}$ decreased in the presence of the EPI. The mutant also showed a 94-fold decrease in sensitivity to ciprofloxacin (McCay et al., 2010).

\section{Real-time PCR}

Changes in expression of the MexA, MexC, MexE, and MexX genes of BC-adapted isolate no. 87 were measured in the absence and presence of a combination of $\mathrm{BC}+$ Ser. (at subinhibitory conc.); expression was compared to that of wild-type strain ATCC 15442 as shown in Table 6.

For untreated BC-adapted isolate no. 87, MexA gene was expressed at a 9.189-fold, MexC 8.5-fold, MexE 5.5-fold, and MexX 4-fold higher than the control sample. These results are in agreement with previous studies which reported that 4- to 10-fold increase in the activity of a mexA (Dumas et al., 2005; Gomaa et al., 2016; Pasca et al., 2012; Srikumar et al., 2000).

In another study by Wolter et al. (2009), it was documented that the expression of mexA gene in PA431 was twofold higher than PAO1, while genes mexC and mexE showed higher expression by about 340- to 375-fold, respectively, in PA30 than in PAO1, and increased three- to four-fold in strain PA431. In another hand, of mexX gene expression in PA431 was higher than PAO1 by 50 -fold and lower in PA30 by two-fold.

McCay et al. (2010) observed that the expression of both mexA and mexC was increased in the $\mathrm{BC}$-adapted isolate. Expression of mexE and mexX in $\mathrm{BC}$-adapted isolate was half that of 10421 in absence of $\mathrm{BC}$, although expression of mexX was increased in $\mathrm{BC}$-adapted isolate than 10421 in presence of $\mathrm{BC}$.

Decreased expression was noticed by the addition of a combination of BC + SER to the growth medium $(256 \mathrm{mg} / \mathrm{l} \mathrm{BC}+700$ $\mathrm{mg} / \mathrm{l} \mathrm{SER}$ ); the expression of MexA, MexC, MexE, and MexX was decreased to 3.9-, 3-, 1.9-, and 1.4-fold, respectively. These results 
Table 5. The FIC index of benzalkonium chloride and sertraline.

\begin{tabular}{|c|c|c|c|c|c|c|}
\hline \multirow[b]{2}{*}{ Isolate } & \multirow{2}{*}{$\begin{array}{l}\text { Type of } \\
\text { biocide }\end{array}$} & \multicolumn{2}{|c|}{ MIC (mg/l) } & \multirow{2}{*}{$\begin{array}{l}\text { FIC of } \\
\text { each }\end{array}$} & \multirow{2}{*}{$\begin{array}{c}\text { FIC } \\
\text { index }\end{array}$} & \multirow[b]{2}{*}{ Outcome } \\
\hline & & Alone & $\begin{array}{c}\text { In } \\
\text { combination }\end{array}$ & & & \\
\hline \multirow{2}{*}{ Isolate no. 87} & $\mathrm{BC}$ & 2,048 & 256 & 0.125 & \multirow{2}{*}{0.92} & \multirow{2}{*}{ Partial synergy } \\
\hline & Ser. & 1,000 & 800 & 0.8 & & \\
\hline
\end{tabular}

$\mathrm{BC}=$ benzalkonium chloride; Ser $=$ sertraline.

Table 6. Expression of MexA, MexC, MexE, and MexX of the BC-adapted isolate no. 87 (untreated and treated).

\begin{tabular}{|c|c|c|c|c|c|c|c|c|c|}
\hline \multirow{2}{*}{ Sample } & \multirow{2}{*}{$\frac{R P S L}{\mathrm{CT}^{1}}$} & \multicolumn{2}{|c|}{$\operatorname{MexA}$} & \multicolumn{2}{|c|}{ MexC } & \multicolumn{2}{|c|}{ MexE } & \multicolumn{2}{|c|}{$\operatorname{MexX}$} \\
\hline & & CT & Fold change $^{2}$ & CT & Fold change & CT & Fold change & CT & Fold change \\
\hline $\begin{array}{c}\text { Control } \\
\text { (ATCC 15442) }\end{array}$ & 12.74 & 21.86 & - & 21.73 & - & 17.59 & - & 18.65 & - \\
\hline $\begin{array}{c}87 \\
\text { (untreated) }\end{array}$ & 13.64 & 19.56 & 9.1896 & 19.54 & 8.5150 & 16.02 & 5.5404 & 17.52 & 4.0840 \\
\hline $\begin{array}{c}87 \\
\text { (Treated) }\end{array}$ & 13.05 & 20.19 & 3.9449 & 20.43 & 3.0525 & 16.94 & 1.9453 & 18.38 & 1.4948 \\
\hline
\end{tabular}

${ }^{1}$ The cycle number at which the fluorescence generated within a reaction crosses the threshold.

${ }^{2}$ The ratio of the target gene expression in the test sample over the control sample.

indicated that $\mathrm{BC}$-adapted isolates overexpressed the four efflux pump genes; and by treatment with sertraline (EPI), the expression of these genes was down-regulated to quarter; so we investigated that the efflux pump was the mainly resistant mechanism to the $\mathrm{BC}$.

\section{CONCLUSION}

In conclusion, the indiscriminate use of $\mathrm{BC}$ in various fields and their accumulation represents a potential risk for selective pressure towards the selection of adapted bacteria, which could be cross-resistant to other disinfectants and antibiotics.

Although, in the present study, efflux pump overexpression seemed to be the main resistance mechanism of $P$. aeruginosa adaptation to $\mathrm{BC}$; other mechanisms of resistance should be studied to combat this phenomenon.

Our approach in this study was to obtain a final product contained BC plus sertraline (efflux pump inhibitor) which can be used pharmaceutically to restore disinfectant activity against BC-adapted $P$. aeruginosa and minimize further developments of resistant strains.

\section{REFERENCES}

Adabi M, Talebi-Taher M, Arbabi L, Afshar M, Fathizadeh S, Minaeian S. Spread of Efflux Pump Overexpressing-Mediated Fluoroquinolone Resistance and Multidrug Resistance in Pseudomonas aeruginosa by using an Efflux Pump Inhibitor. Infect Chemother, 2015; 47: 98-104.

Antonio G, Gadea R, Angel M. Effects of exposure to quaternaryammonium-based biocides on antimicrobial susceptibility and tolerance to physical stresses in bacteria from organic foods. Food Microbiol, 2017; 63:58-71.

Antunes SC, Nunes B, Rodrigues S, Nunes R, Fernandes J, Correia AT. Effects of chronic exposure to benzalkonium chloride in Oncorhynchus mykiss: cholinergic neurotoxicity, oxidative stress, peroxidative damage, and genotoxicity. Environ Toxicol Pharmacol, 2016; 45:115-22.

Bauer AW, Kirby WM, Sherris JC, Turck M. Antibiotic susceptibility testing by a standardized single disk method. Am J Clin Pathol, 1966; 45:493-6.
Bohnert JA, Szymaniak-Vits M, Schuster S, Kern WV. Efflux inhibition by selective serotonin reuptake inhibitors in Escherichia coli. J Antimicrob Chemother, 2011; 66:2057-60.

Buffet-Bataillon S, Tattevin P, Maillard JY, Bonnaure-Mallet M, Jolivet-Gougeon A. Efflux pump induction by Quaternary Ammonium Compounds (QAC) and fluoroquinolone-resistance in bacteria. Future Microbiol, 2016; 11(1):81-92.

Cabot G, Zamorano L, Moyà B, Juan C, Navas A, Blázquez J. Evolution of Pseudomonas aeruginosa antimicrobial resistance and fitness under low and high mutation rates. Antimicrob Agents Chemother, 2016; 60:1767-78.

CLSI. Clinical and Laboratory Standards Institute. Performance Standards for Antimicrobial Susceptibility Testing. Eighteenth Informational Supplement, Wayne, PA. CLSI document m100-s18. p. 28, 2008.

Dumas J, Delden C, Van Perron K., Thilo K. Analysis of antibiotic resistance gene expression in Pseudomonas aeruginosa by quantitative real-time-PCR. FEMS Microbiol Lett, 2006; 254(2):217-25.

Ebrahimi A, Arvaneh Z, Mahzounieh M, Lotfalian S. Antibiotic Resistance Induction by Benzalkonium Chloride Exposure in Nosocomial Pathogens. International Journal of Infection, 2016; 4(2) 1-5.

El-Naggar W, El-Sokkary MA, Barwa R, Abdel-Rhman HS Phenotypic and Genotypic Studies on Antibacterial Efflux Pumps in Pseudomonas. 2011; 1-10 (December 2015).

Esquisabel ABC, Rodríguez MC, Campo-Sosa AO, Rodríguez C, Martínez-Martínez L. Mechanisms of resistance in clinical isolates of Pseudomonas aeruginosa less susceptible to cefepime than to ceftazidime. Clin Microbiol Infect, 2011; 17:1817-22.

Ferreira C, Pereira AM, Pereira MC, Melo LF, Simoes M. Physiological changes induced by the quaternary ammonium compound benzyldimethyldodecylammonium chloride on Pseudomonas fluorescens. J Antimicrob Chemother, 2011; 66:1036-43.

Gnanadhas DP, Marathe SA, Chakravortty D. Biocidesresistance, cross-resistance mechanisms, and assessment. Expert Opin Investig Drugs, 2013; 22:191-206.

Gomaa NI, Metwally L, Attallah MF, Younis S, Kamel N. Measurement of Efflux Pumps Expression by Relative Quantitative RealTime Polymerase Chain Reaction in Multi-Drug Resistant Pseudomonas aeruginosa Isolated from Suez Canal University Hospital. Journal of Molecular Diagnosis and Vaccines, 2016; 11: 1-8. 
Kawamura-Sato K, Wachino J, Kondo T, Ito H, Arakawa Y. Correlation between reduced susceptibility to disinfectants and multidrug resistance among clinical isolates of Acinetobacter species. J Antimicrob Chemother, 2010; 65:1975-83.

Lambert RJW, Pearson J. Susceptibility testing : accurate and reproducible minimum inhibitory concentration (MIC) and non-inhibitory concentration. J Appl Microbiol, 2000; 88(5):784-90.

Langsrud S, Sundheim G, Borgmann-Strahsen R. Intrinsic and acquired resistance to quaternary ammonium compounds in food-related Pseudomonas spp. J Appl Microbiol, 2003; 95:874-82.

Linares JF, López JA, Camafeita E, Juan P, Rojo F, Martínez JL. Overexpression of the multidrug efflux pumps MexCD-OprJ and MexEFOprN is associated with a reduction of type III Secretion in Pseudomonas aeruginosa. J Bacteriol, 2005; 187(4):1384-91.

Lister PD, Wolter DJ, Hanson ND. Antibacterial-resistant Pseudomonas aeruginosa: clinical impact and complex regulation of chromosomally encoded resistance mechanisms. Clin Microbiol Rev, 2009; 22:582-610.

Livermore DM. Multiple mechanisms of antimicrobial resistance in Pseudomonas aeruginosa: Our worst nightmare? Clin Infect Dis, 2002; 34:634-40.

Lomovskaya O, Lee A, Hoshino K, Ishida H, Mistry A, Warren MS. Use of a genetic approach to evaluate the consequences of inhibition of efflux pumps in Pseudomonas aeruginosa. Antimicrob Agents Chemother, 1999; 43:1340-6.

Mahon CR, Lehman DC, Manuselis G. Textbook of diagnostic microbiology, Fifth edition. USA: W.B. Saunders Company. 2014.

Martins M, McCusker MP, Viveirosb M, Coutoc I, Fanninga S, Pagèsb JM, Amaralb L. A simple method for assessment of MDR bacteria for over-expressed efflux pumps. Open Microbiol J, 2013; 7:72-82.

Mazzola PG, Jozala AF, Novaes LC, Moriel P, Penna TC Minimal inhibitory concentration (MIC) determination of disinfectant and/ or sterilizing agents. Brazilian J Pharm Sci, 2009; 45:241-8.

McCay PH, Ocampo-Sosa AA, Fleming GT. Effect of subinhibitory concentrations of benzalkonium chloride on the competitiveness of Pseudomonas aeruginosa grown in continuous culture. Microbiology, 2010; 156:30-8.

NCCLS. National committee for clinical laboratory standards "Performance standards for antimicrobial susceptibility testing: Fourteenth informational supplement m100-s14." NCCLS, Wayne, PA, 2004.

Nemat-gorgani N. Detecting mutations related to antibiotic resistance in Pseudomonas aeruginosa. Master thesis, San Jose State University, 2009.

Nguyen KV, Nguyen TV, Nguyen HTT, Van Le D. Mutations in the GyrA, ParC, and mexR genes provide functional insights into the fluoroquinolone-resistant Pseudomonas aeruginosa isolated in Vietnam. Infect Drug Resist, 2018; 275-82.

Nones J, Savi GD, Müller L, Trentin AG. A Brazilian Bentonite Previously Treated with Benzalkonium Chloride Reduces Pseudomonas aeruginosa Growth. Journal of Bacteriology and Mycology, 2017; 4(2): 1-7.

Pasca MR, Dalla Valle C, Jesus Lopes Ribeiro AL, Buroni S, Papaleo MC, Bazzini S, Udine C, Incandela ML, Daffara S, Fani R, Riccardi G. Evaluation of fluoroquinolone resistance mechanisms in Pseudomonas aeruginosa multidrug resistance clinical isolates. Microb Drug Resist, $2012 ; 18: 23-32$.
Poonsuk K, Chuanchuen R. Detection of the mex efflux pumps in Pseudomonas aeruginosa by using a combined resistance-phenotypic markers and multiplex RT-PCR. Open J Med Microbiol, 2014; 153-60.

Rakic-Martinez M, Drevets DA, Dutta V, Katic V, Kathariou S Listeria monocytogenes strains selected on ciprofloxacin or the disinfectant benzalkonium chloride exhibit reduced susceptibility to ciprofloxacin, gentamicin, benzalkonium chloride, and other toxic compounds. Appl Environ Microbiol, 2011; 77:8714-21.

Satish KP, Robert CMJ, George ME. Antimicrobial combinations. In: Lorian (Ed.). Antibiotics in laboratory medicine. Philadelphia: Lippincott Williams \& Wilkins, pp. 366-425, 2005.

Savov E, Trifonova A, Todorova I, Gergova I, Borisova M, Ananieva M, Kjoseva E, Kardjeva V. Assesment of the resistance of clinical isolates Pseudomonas aeruginosa to quinolones. Trakia J Sci, 2014; $12: 221-6$.

Srikumar R, Paul CJ, Poole K. Influence of mutations in the mexR repressor gene on expression of the MexA-MexB-OprM multidrug efflux system of Pseudomonas aeruginosa. J Antimicrob Chemother, 2000; 46:885-93.

Suman G, Khan M, Sabitha K, Jamil K. Mutation in mexR -gene leading to drug resistance in corneal keratitis in human. Indian J Exp Biol, 2006; 44:929-36.

Vaez H, Faghri J, Isfahani BN, Moghim S, Yadegari S, Fazeli H. Efflux pump regulatory genes mutations in multidrug resistance Pseudomonas aeruginosa isolated from wound infections in Isfahan hospitals. Adv Biomed Res, 2018; 3.

Vijaya K, Saleh Al-Aboody M, Alfonaisan MK, Sandle T. In vitro susceptibility of multidrug resistant Pseudomonas aeruginosa clinical isolates to common biocides. Int J Res Pharm Sci, 2016; 7:110-6.

Walsh SE, Maillard J, Russell AD, Catrenich CE, Charbonneau DL, Bartolo RG. Development of bacterial resistance to several biocides and effects on antibiotic susceptibility. J Hospital Infect, 2003; 6701:98-107.

Wiegand I, Hilpert K, Hancock REW. Agar and broth dilution methods to determine the minimal inhibitory concentration (MIC) of antimicrobial substances. Nat Protoc, 2008; 3:163-75.

Wolter DJ, Black JA, Lister PD, Hanson ND. Multiple genotypic changes in hypersusceptible strains of Pseudomonas aeruginosa isolated from cystic fibrosis patients do not always correlate with the phenotype. J Antimicrob Chemother, 2009; 64(2):294-300.

Yuan J, Reed A, Chen F, Stewart CN. Statistical analysis of realtime PCR data. BMC Bioinform, 2006; 7:85.

Yuruken Z, İseri L, Ünaldi Ö, Durmaz R. Analysis of crosstransmission and antimicrobial resistance of Pseudomonas aeruginosa and Acinetobacter baumannii isolates causing nosocomial infection in an intensive care unit. Turkiye Klin J Med Sci, 2016; 36:1-6.

How to cite this article:

Osman ME-S, El-Hendawy HH, Abd El-All SM, Hassan AM, Mahmoud DE. Correlation between resistance of Pseudomonas aeruginosa to benzalkonium chloride and expression of efflux pump genes. J Appl Pharm Sci, 2018; 8(12): 044-052. 